\title{
A perching mechanism for micro aerial vehicles
}

\author{
Mirko Kovač • Jürg Germann • Christoph Hürzeler • \\ Roland Y. Siegwart • Dario Floreano
}

Received: 25 November 2009 / Revised: 21 January 2010 / Accepted: 1 April 2010 / Published online: 4 May 2010

(C) Springer-Verlag 2010

\begin{abstract}
Micro Aerial Vehicles (MAVs) with perching capabilities can be used to efficiently place sensors in aloft locations. A major challenge for perching is to build a lightweight mechanism that can be easily mounted on a MAV, allowing it to perch (attach and detach on command) to walls of different materials. To date, only very few systems have been proposed that aim at enabling MAVs with perching capabilities. Typically, these solutions either require a delicate dynamic flight maneuver in front of the wall or expose the MAV to very high impact forces when colliding
\end{abstract}

Electronic supplementary material The online version of this article (doi:10.1007/s12213-010-0026-1) contains supplementary material, which is available to authorized users.

M. Kovač $(\varangle)$ · J. Germann · D. Floreano

Ecole Polytechnique Fédérale de Lausanne (EPFL), Laboratory of Intelligent Systems (LIS),

1015 Lausanne, Switzerland

e-mail: mirko.kovac@epfl.ch

URL: http://lis.epfl.ch

J. Germann

e-mail: jurg.germann@epfl.ch

D. Floreano

e-mail: dario.floreano@epfl.ch

C. Hürzeler · R. Y. Siegwart

Eidgenössische Technische Hochschule Zürich (ETHZ),

Institut für Robotik und Intelligente Systeme (ASL),

8092 Zürich, Switzerland

URL: http://www.asl.ethz.ch

C. Hürzeler

e-mail: christoph.huerzeler@mavt.ethz.ch

R. Y. Siegwart

e-mail: rsiegwart@ethz.ch head-first with the wall. In this article, we propose a $4.6 \mathrm{~g}$ perching mechanism that allows MAVs to perch on walls of natural and man-made materials such as trees and painted concrete facades of buildings. To do this, no control for the MAV is needed other than flying head-first into the wall. The mechanism is designed to translate the impact impulse into a snapping movement that sticks small needles into the surface and uses a small electric motor to detach from the wall and recharge the mechanism for the next perching sequence. Based on this principle, it damps the impact forces that act on the platform to avoid damage of the MAV. We performed 110 sequential perches on a variety of substrates with a success rate of $100 \%$. The main contributions of this article are (i) the evaluation of different designs of perching, (ii) the description and formal modeling of a novel perching mechanism, and (iii) the demonstration and characterization of a functional prototype on a microglider. (See accompanying video and http://lis.epfl.ch/microglider/perching.mpg.)

\section{Introduction}

Efficient sensor placement is of crucial importance for distributed sensor networks, and is necessary for a variety of different scenarios [5, 25,37]. The challenge is how to place a large number of sensors in aloft locations where they can well monitor the environment. In order to do that, one possible solution is to equip small MAVs with sensors and the ability to perch to natural and man made structures. As perching for MAVs we define the ability to attach to inclined surfaces or elevated positions out of flight and detach on command. To date, only very 
few systems have been demonstrated recently that can attach to surfaces and only one system has been shown to be able to attach to and detach from vertical surfaces. The main difficulty in the design of such a MAV is that it has to attach to the surface out of flight and subsequently detach from it to allow a reuse of the system or to change its location. All this needs to be achieved while maintaining small size and light weight to be implemented on a MAV.

In this article, we present the development and characterization of a working $4.6 \mathrm{~g}$ perching mechanism. It allows a small MAV to attach head-first to vertical surfaces out of flight and detach from them again on command using a remote control. To fit the perching mechanism to different MAVs with different masses and flight velocities and to reduce the impact forces on attachment, we present a mathematical model that allows to dimension critical components of the mechanism. As a test bed to demonstrate the perching mechanism being successfully integrated on a MAV, we mount it on a microglider which is a slightly bigger version of the system presented in [20] and uses components of the commercially available MicroCeline [38].

Different approaches have been presented to date that tackle the challenge of perching for MAVs. Anderson et al. [1] recently presented a variety of different perching concepts where the best solution consists of a small propelled MAV with a mass between 42 and $510 \mathrm{~g}$ that crashes into the surface at stall speed and adheres to it using liquid glue. It then hangs down on a theater and uses a razor blade to cut the threat to free itself and retake flight. Although this is a very simple and innovative design, its main limitation is that the perching can be repeated only as many times as many sticky pads are integrated on the airplane. Ideas are described how to store more than one sticky pad, but have not been implemented yet. The paper mentions that it can perch successfully, but no systematic characterization of the perching capabilities have been presented so far. Like other glue based attachment principles, this approach may as well not work on wet or dirty surfaces. Also is the 'rat-glue' used on the presented prototype in liquid state and detaches by itself after $60 \mathrm{~min}$, limiting the perching time. Depending on the MAV robustness, this approach may as well be limited to very light weight or slowly flying MAVs as the impact forces on crash with the wall are directly translated to the structure of the MAV. To reduce the risk for structural damage, damping devices or flight maneuvers would be required to reduce the impact velocity.

Another project has been recently presented by Lussier-Desbiens and Cutkosky [24] where a glider is flying towards a wall, stalls and attaches to the wall using microspines. To do this autonomously, it incorporates an ultrasonic distance range sensor, a complete Paparazzi autopilot and suspension which is covered with microspines, similar to the ones used in [2]. Although this realization can attach to a variety of materials exploiting the surface asperity, it requires a relatively delicate dynamic stall maneuver of the entire MAV and adequate control, which reduces the success rate of attachment to $80 \%$ in the current early stage prototype. Detachment has not been demonstrated yet but could include concepts such as jumping off the surface or using propellers to reinitiate flight. The attachment has been demonstrated on a comparably heavy glider of $400 \mathrm{~g}$, which flies relatively fast at $9 \mathrm{~m} / \mathrm{s}$. For smaller MAV, such as the very slowly flying and light weight microglider used in this paper, stalling maneuvers may be even more delicate due to the low Reynolds number flight regime [27] which is difficult to control. As well do small or indoor flying MAVs impose strict weight requirements that do not allow the integration of heavy sensors or complex computation [38].

A similar concept has been described by Cory and Tedrake [6] who presented a glider that can successfully and precisely land on a string using a hook as the landing gear. To be able to do this, the glider is tracked in $3 \mathrm{D}$ in a lab environment using a VICON system with six cameras and is controlled off-board. Wickenheiser and Garcia [35] aim at developing a perching aircraft that changes its tail configuration to decelerate in front of a vertical surface and attach to it. So far, the project has focussed mainly on aerodynamics and control and not on the attachment itself. Bayraktar and Feron [4] recently presented a helicopter that can land on inclined surfaces of up to $60^{\circ}$ using velcro on its landing gear. Analogous to the previous two systems, this helicopter is tracked and controlled externally and has not been shown to be able to detach by itself after landing. Wright and Lind [37] are investigating sensor placement using a small MAV with morphing wings. The work so far has addressed the computational analysis of the aerodynamics of landing on a vertical surface without integrating mechanisms that would allow it to actually attach to it. Roberts et al. [31] recently presented a hovering platform that can autonomously attach to and detach from ceilings using actuated magnets. Its limitation to date is that it only works on horizontal and ferromagnetic ceilings.

Numerous other projects deal with the challenge of attachment and detachment from the perspective of applying it to climbing robots. All these systems however are not designed to fly and they have the tendency to 


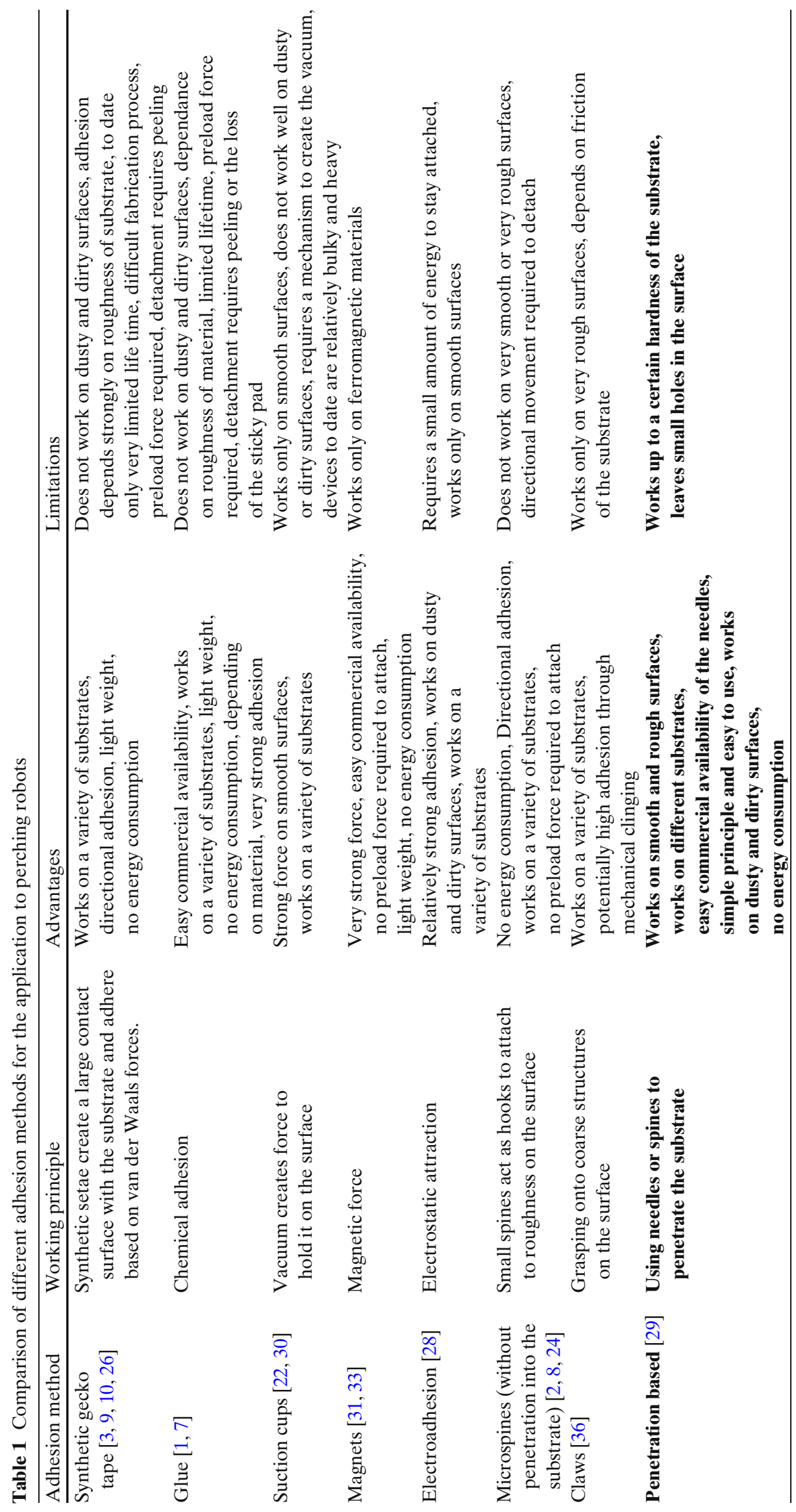


Table 2 Hardness of some prevalent man-made and natural substrates to which our perching mechanism can attach to

\begin{tabular}{ll}
\hline Substrate & Hardness [ShoreD] \\
\hline Balsa wood & 24 \\
Poplar bark & 36 \\
Poplar wood & 54 \\
Okuome wood & 61 \\
Wallpaper & 62 \\
Spruce wood & 65 \\
Birch wood & 69 \\
Composite hardboard wood & 76 \\
MDF & 87 \\
Painted concrete facade & 89 \\
\hline
\end{tabular}

be relatively slow and heavy, which limits their applicability to MAVs. The interested reader may be referred to $[2,26,28,32,36]$ for an overview of existing state of the art robots that use different climbing techniques.

Several different adhesion methods, such as synthetic gecko tape, suction cups, magnets, needles etc., could be used to attach to the surface. In Table 1 we provide a summary evaluation of the advantages and limitations of the different methods that has been presented in the literature to date. We decided to base our design on penetration based clinging using needles due to its applicability for a wide range of prevalent surface materials, its ease of use and utility on smooth as well as rough surfaces.

As we will see later, our perching mechanism works successfully on vertical surfaces with a hardness between 24ShoreD and 89ShoreD. This range corresponds to materials such as tree barks, different kinds of wood, facades of buildings and painted concrete. Examples of the hardness of different prevalent manmade and natural materials where our mechanism can perch on are listed in Table 2.

In the following sections we will first illustrate the process and design criteria that we applied for designing our mechanism. We will then present the modeling that allows to dimension the parts of the mechanism and describe the mechanical implementation. Finally, we will characterize the perching mechanism and demonstrate its integration with a microglider to successfully attach to walls of different materials and detach on command using a remote control. Video footage of its behavior indoors and outdoors can be seen in the accompanying video material. ${ }^{1}$

\footnotetext{
$\overline{{ }^{1} \text { See accompanying video and http://lis.epfl.ch/microglider/ }}$ perching.mpg.
}

\section{Design}

In order to design our perching mechanism, we assume that it will be mounted on the tip of a MAV which is flying at a constant forward velocity towards a vertical wall. Our perching mechanism has to fulfill two functions, i.e. autonomous attachment and detachment. In this section, we propose and discuss different design principles to fulfill these functions.

As the perching mechanism will be mounted on a MAV and has to work robustly, we define the design requirements to be (i) light weight, (ii) small size, (iii) effectiveness, (iv) structural simplicity and (v) exertion of little force on the MAV when perching to the wall. In line with these requirements, we will choose the design principles to implement.

\subsection{Surface attachment}

For the attachment to the surface, we considered three attachment principles (Fig. 1) and compare them using a standard engineering qualitative comparison as proposed in e.g. [34]. The first principle (A) consists of two needles (representing a symmetrical array of one or more needles) that are mounted in front of the glider and act like darts to stick to the surface. Since there are no moving or flexible parts required to implement this principle, it is very light weight, small and simple. Its drawback is that it is not very effective as it can stick only well if the impact of the MAV is exactly in line with the needles. An advantage of this principle is that the entire impact impulse is used to stick the needles into the surface. However, the deceleration distance is given by the protrusion depth of the needles, which is typically very short and therefore leads to very high forces acting on the structure of the MAV. For example, a very light weight MAV of $20 \mathrm{~g}$ that is flying at $4 \mathrm{~m} / \mathrm{s}$ and sticks to the wall with a protrusion depth of the needles of $1 \mathrm{~mm}$ would experience an average impact force of $160 \mathrm{~N}$, which is unacceptable for the structure of such a small MAV. Possibilities to deepen the protrusion depth by using different needles as a function of the substrate where the MAV shall attach to are very limited and would significantly add complexity to the system.

The second principle (B) consists of a grasping mechanism that is extended when the MAV impacts the surface and grasps subsequently, due to its elasticity, into the surface. This solution has the advantage that it can adapt well to the surface structure as the grippers extend first and then slide along the surface to grasp. The impact of the MAVs is first damped and the preloaded flexible arms then push the needles into the 
Fig. 1 Attachment principle for the perching mechanism. A sticks to the surface like a dart, $\mathbf{B}$ grasps to stick the needles into the surface,

C snaps the arms to stick the needles into the surface
(A)

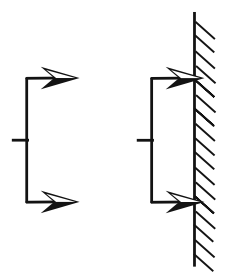

(B)

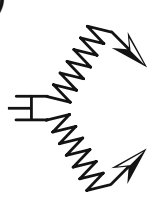

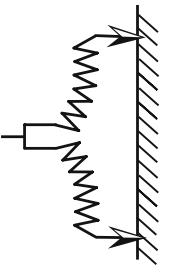

(C)

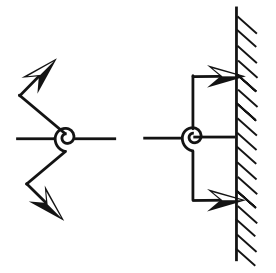

surface. This principle therefore exerts little forces on the structure of the MAV, but makes the penetration less effective due to the losses during damping.

The third and final principle that we considered (C) consists of two arms that are charged using a torsion spring. Once the MAV impacts the surface, the energy in the spring is released by a mechanical trigger and the two arms snap forward to stick the needles into the surface. Compared to the first two principles, (C) is more effective as the force that acts on the needles when the mechanism snaps can be adapted by using a different spring or mass of the arms. Depending of the arm length, the deceleration distance can easily be adjusted, which allows to keep the forces acting on the structure of the MAV low. The main drawback is that it requires the integration with a torsion spring which makes it structurally less simple compared to principle (A). Based on the comparison of these three possible attachment principles as qualitatively summarized in Table 3, we decided to use principle (C) for the attachment of our perching mechanism.

\subsection{Detachment and recharging}

Based on the surface attachment principle (C) we considered several possibilities to pull the arms backward to detach and recharge the mechanism for the next perching sequence. Ways to achieve this include small commercially available servos [13], Shape Memory Alloy (SMA) based actuators [20] or a small DC pager motor with a custom designed gearbox. The main advantage of the latter mechanism compared to the others is that it allows to dimension the motor and

Table 3 Qualitative evaluation summary of the three working principles for the attachment of our perching mechanism (Fig. 1) (- -: Very unfavorable, 0 : Neutral, ++ : Very favorable)

\begin{tabular}{llll}
\hline Criteria & $(\mathrm{A})$ & $(\mathrm{B})$ & (C) \\
\hline Weight & ++ & 0 & + \\
Size & + & - & $\mathbf{0}$ \\
Effectiveness & - & - & ++ \\
Simplicity & + & - & $\mathbf{0}$ \\
Structural strain on MAV & -- & + & ++ \\
\hline
\end{tabular}

gearbox exactly to the needs of the perching mechanism which makes it a lighter solution than the commercially available servos. Compared to the SMA actuators, it is more effective as it avoids the small actuation length and hysteresis effects of SMAs.

We therefore decide to implement a DC motor actuated coil to pull back two strings that are attached to the arms. Once the arms are pulled back, a small magnet fixes them in their charged position. In case that the detachment would not succeed immediately, this mechanism could decharge and recharge again several times to pull the needles out of the wall.

\section{Modeling of the perching mechanism}

In this section we model the kinematics of the perching mechanism in order to dimension it for a given MAV. The goal is to dimension the torsion spring and the mass of the arms in a way that the MAV is decelerated while the arms are snapping forward and has zero velocity in the moment when the needles penetrate into the surface. This is a necessary condition to avoid that the MAV crashes into the surface or that the snapping would bounce it off the surface, both which would not allow a controlled and efficient perching to the substrate. Mathematically expressed, this requires that the impulse of the MAV is equal to the impulse generated by the snapping of the arms (Fig. 2). For these calculations we assume that (i) the only mass of the arms is a point mass on its tips, (ii) the only contribution to the deceleration of the MAV is the snapping movement and (iii) that the spring force is perfectly linear with angular deflection.

$$
p_{x}(t)=m_{\text {mav }} v_{\text {mav }}, \quad t=t_{0}
$$

$p_{x}$ is the impulse of the entire system in the horizontal flying direction. $m_{\text {mav }}$ and $v_{\text {mav }}$ are the mass and velocity of the MAV. $t_{0}$ is the moment in flight before the trigger touches the substrate.

After time $t_{1}$, when the trigger touched the substrate and initiated the snapping, impulse is generated by the moving arms, each having a mass $m_{a}$, and the MAV is 


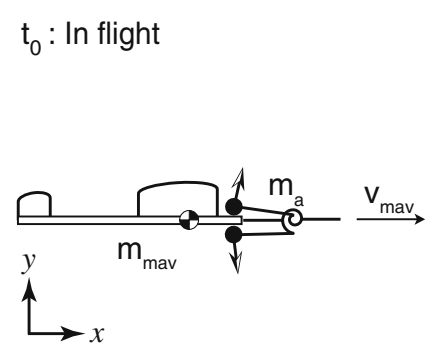

Fig. 2 Kinematic model of the perching mechanism mounted on a MAV. $m_{\text {mav }}$ mass of the MAV, $v_{\text {mav }}$ velocity of the MAV in x-direction, $m_{a}$ mass of one arm, $v_{a}$ velocity of the arms in $\mathrm{x}$-direction. $t_{0}$ is the moment in flight before impacting on the

decelerated by the velocity $-v_{d}$ to satisfy the impulse balance.

$$
p_{x}(t)=m_{\text {mav }} v_{\text {mav }}+2 m_{a} v_{a}-m_{\text {mav }} v_{d}, \quad t_{1}<t<t_{2}
$$

We dimension the mass $m_{a}$ and the snapping velocity $v_{a}$ in a way that the MAV is decelerated to zero velocity right before the time $t_{2}$ when the needles penetrate the substrate. In order to do this, we draw an energy balance and calculate the angular velocity $\omega(t)$ of the arms relatively to the MAV.

$$
\begin{array}{ll}
\frac{1}{2} c_{s} \varphi(t)^{2}=-2 \cdot \frac{1}{2} J \omega(t)^{2}, & t_{1}<t<t_{2} \\
\omega(t)=\sqrt{\frac{c_{s}}{2 J}\left(\varphi\left(t_{0}\right)^{2}-\varphi(t)^{2}\right)}, & t_{1}<t<t_{2}
\end{array}
$$

With $c_{s}$ being the spring constant of the torsion spring, $\varphi$ the opening angle of the two arms, and $J$ the moment of inertia of the two arms. Based on this, we can derive the velocity of the weights in $\mathrm{x}$-direction $v_{a}$ relative to the MAV.

$v_{a}(t)=l_{s} \omega(t) \cos \left(90-\frac{\varphi(t)}{2}\right), \quad t_{1}<t<t_{2}$

$l_{s}$ is the length of the arms. Applying the parallel axis theorem $J=m_{a} l_{s}^{2}$ and giving the condition that the MAV has zero velocity when the needles penetrate the substrate $v_{\text {mav }}\left(t_{2}\right)=0$, we obtain the relation between the mass on the arms and the torsion spring.

$m_{a} c_{s}=\frac{1}{2} \cdot \frac{v_{\text {mav }}^{2} \cdot m_{\text {mav }}^{2}}{\varphi\left(t_{0}\right)^{2}-\varphi\left(t_{2}\right)^{2}}$

In order to maintain the impulse balance, we could therefore either use a smaller spring and a larger mass of the arms, or combine a stronger spring and a smaller mass of the arms. Using a large mass of the arms or surface, $t_{1}$ the moment when the trigger touches the surface and initiates the snapping movement, $t_{2}$ moment when the needles at the tip of the arms touch the surface

a stronger spring may both increase the mass of the entire system. The goal is now to find the optimum in this trade-off and to chose a combination of spring and mass of the arms to keep the weight of the perching mechanism as low as possible. For simplicity we assume here that the gearbox and motor to charge the springs are identical for all the different combinations.

To determine the relationship between the spring mass $m_{s}$ and the spring constant $c_{s}$, we measure the mass of ten standard steel torsion springs with a spring constant between $0.0137 \mathrm{~N} \mathrm{~mm} /{ }^{\circ}$ and $4.66 \mathrm{~N} \mathrm{~mm} /{ }^{\circ}$ that are commercially available at [12] (Fig. 3). Performing a least square linear fit on these ten points, we find the relation $m_{s}=1.1 \cdot c_{s}+0.014$. Based on this relation and (6), we can plot the trade-off between the spring constant, the impulse of the MAV and the sum of the masses of the arms and the spring (Fig. 4).

Our microglider test bed, with an entire system mass of $5 \mathrm{~g}$ and a flight velocity of $2 \mathrm{~m} / \mathrm{s}$ has an impulse of

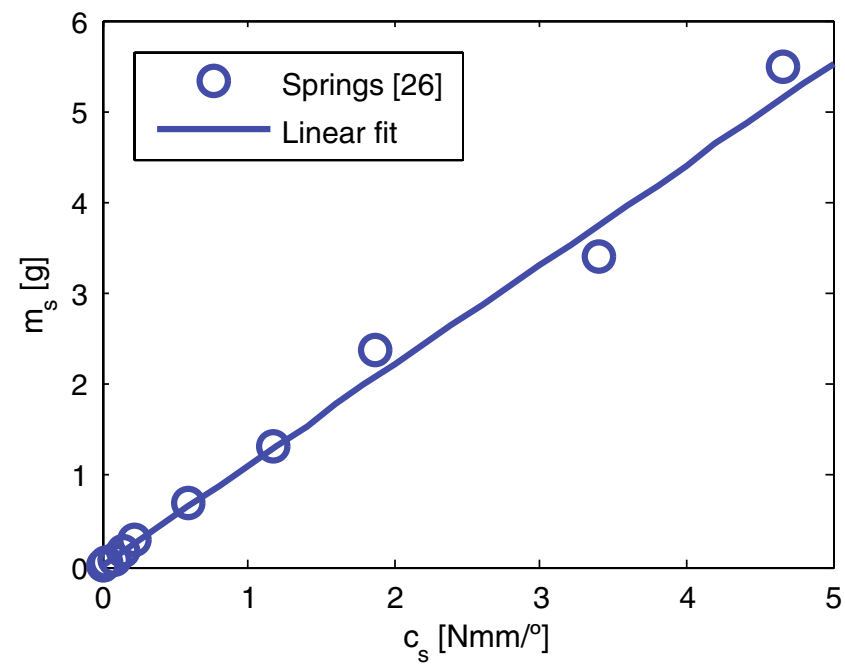

Fig. 3 Relation between the spring mass $m_{s}$ and the spring constant $c_{s}$ for a set of ten commercially available springs [12] 

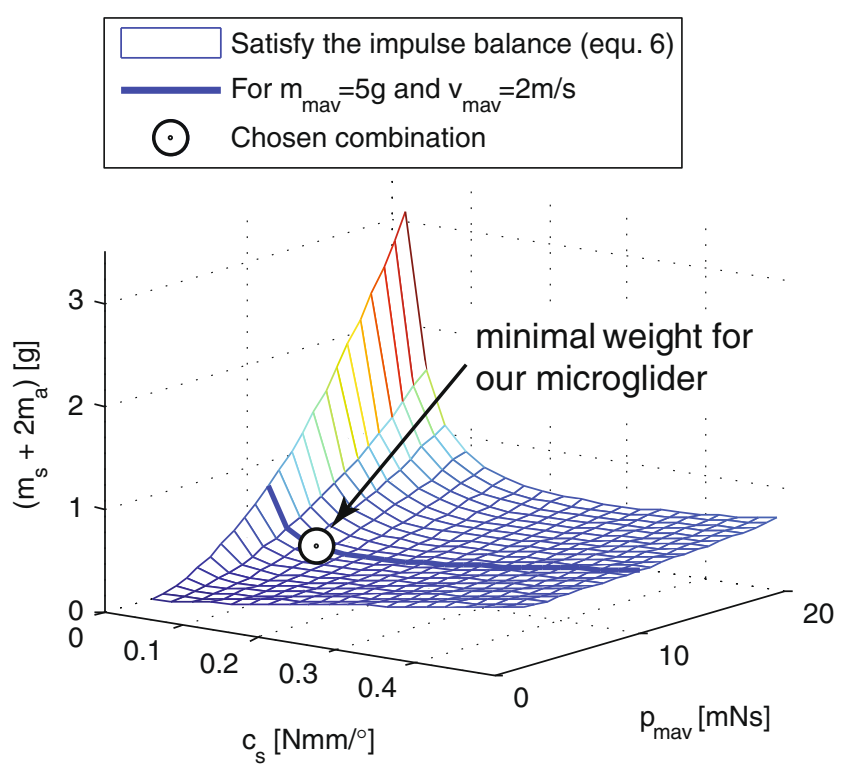

Fig. 4 The sum of the masses of the spring $m_{s}$ and the arms $m_{a}$ versus the impulse of the MAV $p_{\text {mav }}$ and the spring constant $c_{S}$ of the torsion spring. For a microglider with a mass of $5 \mathrm{~g}$, flying at $2 \mathrm{~m} / \mathrm{s}$, we choose the combination with minimal mass for the entire system (indicated with a 'o')

$10 \mathrm{mN} \mathrm{s}$. We therefore choose a spring with a spring constant of $0.0854 \mathrm{~N} \mathrm{~mm} /{ }^{\circ}$ and a mass of $0.15 \mathrm{~g}$ at the tips of each arm to satisfy the required conditions and minimize the weight of the entire perching mechanism. The best fit and resulting configuration is highlighted with a 'o' in Fig. 4.

\section{Mechanical design and implementation}

As next step, we implemented the design principles as described above in Computer Aided Design (CAD) and fabricated the prototype. The details of the mechanical realization can be seen in the CAD view and the prototype in Fig. 5a-c, along with a picture of the realized prototype in Fig. 5d-e.

Realized is this perching sequence through a trigger (Fig. 5d(a)) with a small linear spring on its end to push it back in position. When the trigger touches the surface, it separates the magnets (Fig. 5b(b)), which allows the torsion spring (Fig. $5 \mathrm{~d}(\mathrm{c})$ ) to snap its arms (Fig. 5d(d)) forward and stick the needles (Fig. 5d(e)) into the surface.

In order to satisfy the impulse balance, we add small weights (Fig. 5d(f)) to each of the arms. In order to detach and recharge the mechanism, a small DC motor (Fig. 5a(g)) and gearbox (Fig. 5a(h)) pulls the arms backwards and positions the magnets that are mounted on the string (Fig. 5a(i)) so that they keep the mecha- nism charged. The structural stability of the mechanism is ensured by using a stabilization bar (Fig. $5 a(j))$. In order to keep the MAV in a predefined position when perched to the wall, we add a support rod (Fig. $5 \mathrm{a}(\mathrm{k})$ ) to the mechanism. The carbon rod (Fig. $5 \mathrm{a}(\mathrm{m})$ ) ensures a stable position to be able to detach from the wall. The infra red receiver with battery (Fig. $5 \mathrm{c}(\mathrm{n})$ ), which is mounted on the gearbox, allows to remote control the mechanism to detach and recharge.

The attachment sequence is illustrated in Fig. 6. In flight, the perching mechanism is in a charged state (Fig. 6a). Once it touches the surface, the trigger separates the magnets (Fig. 6b) and the arms snap forward and stick the needles into the substrate (Fig. 6c). Finally, the mechanism settles in its stable position on the surface (Fig. 6d) and is then ready to detach from it on command to reinitiate flight.

Using the spring as calculated for the microglider, we need a force of $5.3 \mathrm{~N}$ to recharge the perching mechanism. The smallest low cost motor that we found is a $4 \mathrm{~mm}$ DC pager motor, which provides a torque of $0.038 \mathrm{mN} \mathrm{m}$ at a motor speed of $8,000 \mathrm{t} / \mathrm{min}$. This motor needs a gearbox with a transmission ratio of at least 178 to achieve the recharging. The implemented custom designed gearbox has four stages with a total transmission ratio of 833 . This means that it provides a security factor of 4.6, which should be sufficient.

The two magnets that hold the mechanism in the charged position are commercially available NeodymeFer magnets with a diameter of $2 \mathrm{~mm}$ and a pulling force of $1 \mathrm{~N}$. The spring which pushes back the trigger to its position has a spring constant of $0.09 \mathrm{~N} \mathrm{~mm}$. At an acting distance of $2 \mathrm{~mm}$ it exerts a force of $0.18 \mathrm{~N}$. This is high enough to push the trigger back after impact to its initial position, but does not significantly contribute to the deceleration of the MAV. In order to estimate its influence on the deceleration, we can calculate the impact force of a MAV to the wall. The energy of movement is defined as $E_{k i n}=(1 / 2) \cdot m_{\text {mav }} v_{\text {mav }}^{2}$. For our rather light weight microglider, the kinetic energy in flight is $10 \mathrm{~mJ}$. Assuming a deceleration distance of $2 \mathrm{~mm}$, the force acting on the trigger and this spring when touching the surface is $5 \mathrm{~N}$, which is 28 times more than the force provided by the spring. This is the case for our light weight and slow flying microglider. For heavier or faster MAVs the influence would be even less. As implied in the modeling section, it is therefore justified to assume that the impulse of the snapping arms is the major contribution in the deceleration of the MAV. The needles that we use are commercially available steel sawing needles with a diameter of $0.5 \mathrm{~mm}$. The infrared remote control to control the motor is a commercial unit purchased at [11]. 
Fig. 5 Illustration of the details of the mechanical realization in CAD and on the realized prototype.

A Entire perching mechanism, B close up view of the release mechanism where the trigger separates the magnet to allow the arms to snap forward,

C close up view of the front part of the mechanism,

D close up photograph of the gearbox $\mathbf{E}$ photograph of the entire prototype
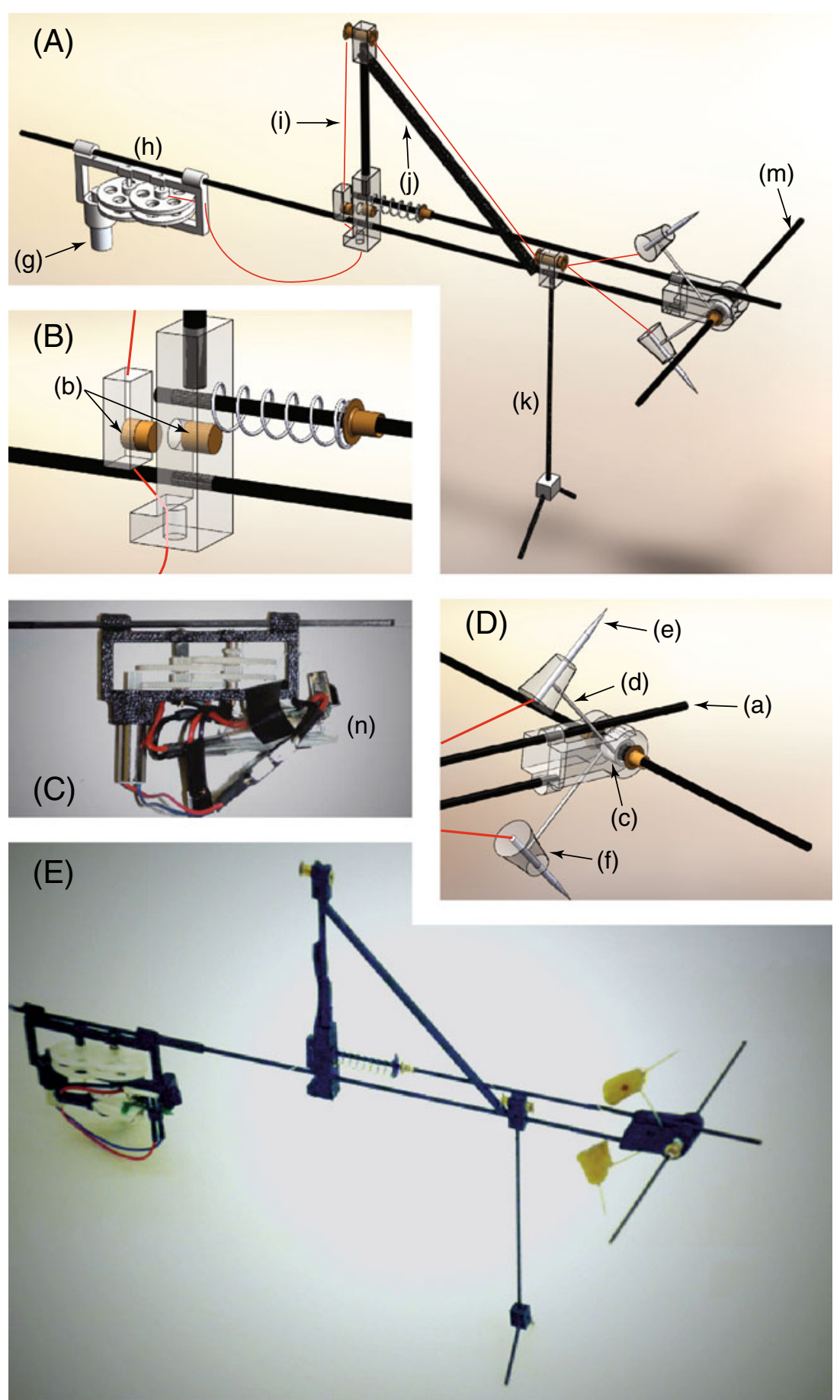

To keep the weight of the system as low as possible while ensuring strength, we use carbon for the trigger and the structural parts and print the connecting pieces and the gearbox out of ABS plus, using a 3D printer [15]. The gears are commercially available and made out of Polyoxymethylene (POM). The mechanical properties of these materials are listed in Table 4. The weight budget is summarized in Table 5 .

\section{Results}

In order to characterize our perching mechanism, we perform three sets of experiments to (1) evaluate how well the perching mechanism attaches to different substrates, (2) test if our modeling of the perching mechanism is correct and (3) characterize the reliability of attachment and detachment on different substrates. We 
Fig. 6 Illustration of the attachment sequence. The grey circles indicate the areas of action. A Charged state in flight, $\mathbf{B}$ the trigger touches the surface and separates the two magnets, $\mathbf{C}$ the arms snap forward and stick the needles into the substrate, $\mathbf{D}$ the mechanism settles in a stable position and is ready to be recharged and detach
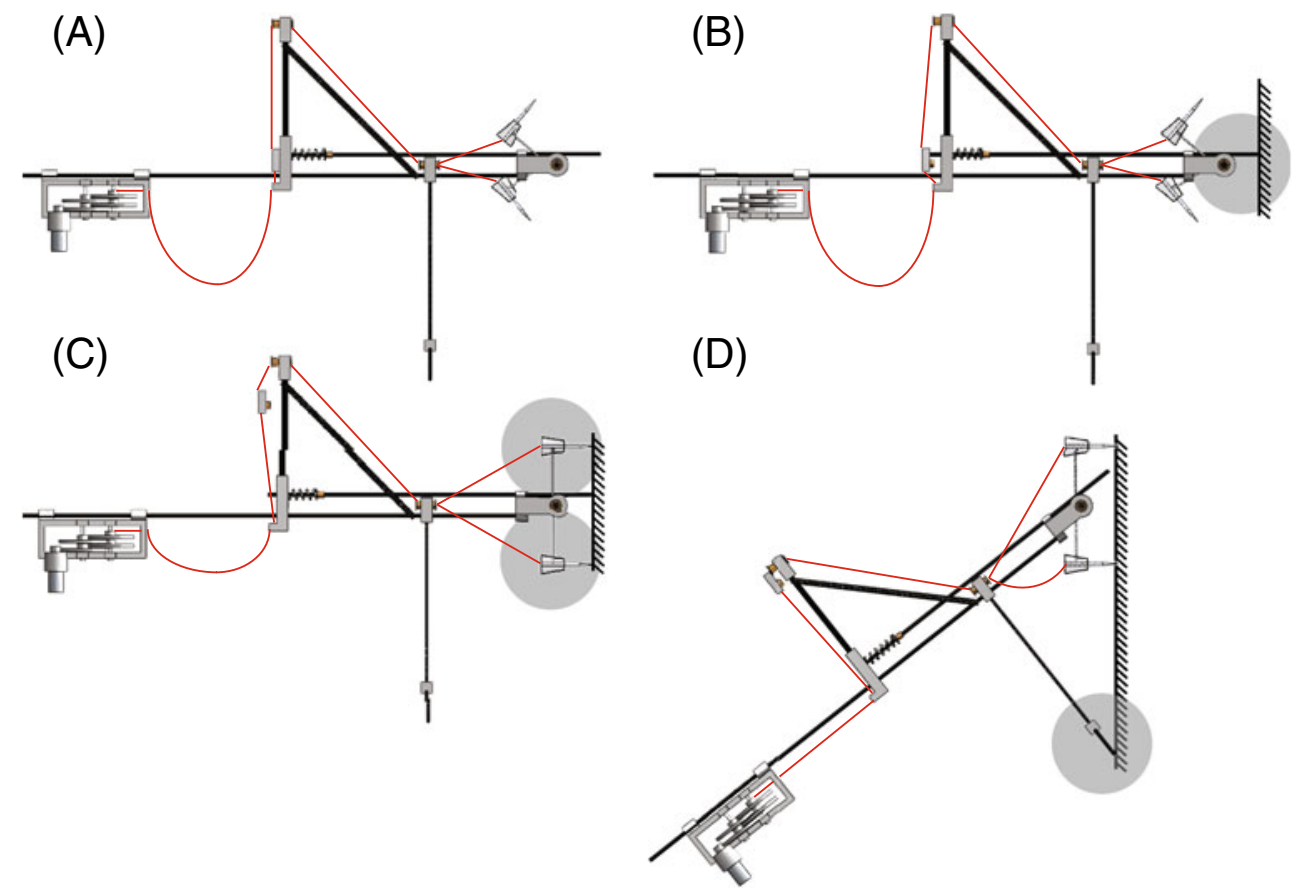

add lead weights to the perching mechanism to simulate the mass of the MAV and launch it using a small linear catapult towards a wall (experimental setup in Fig. 7). Using this setup, we film the perching sequence with a high speed camera [14] at 1,000 Hz. The shape and size of the needles that are used to attach have an influence on the attachment strength [29]. In order to keep this parameter constant, we sharpen the tips of the needles at an angle of $5^{\circ}$ using a metal grinder. After ten launches, we replace the needles to avoid potential effect of wear.

\subsection{Attachment security factor}

In order to evaluate how well the perching mechanism attaches to different natural and man-made substrates, we launch the mechanism to four different substrates, i.e. a painted concrete facade, composite hardboard wood, poplar wood and poplar bark. By incrementally adding weights of $0.5 \mathrm{~g}$ to the attached perching mechanism, we measure the weight that it can hold until it is

Table 4 Properties of the materials used

\begin{tabular}{lcccc}
\hline & $\begin{array}{c}\text { Alu } \\
7,075\end{array}$ & POM & $\begin{array}{c}\text { Carbon } \\
\text { prepreg }\end{array}$ & $\begin{array}{c}\text { ABS } \\
\text { plus }\end{array}$ \\
\hline Density $\left[\mathrm{g} / \mathrm{cm}^{3}\right]$ & 2.7 & 1.56 & 1.55 & 1.04 \\
E-Module $[\mathrm{GPa}]$ & 69 & 5.2 & 130 & 2.2 \\
Yield strength $[\mathrm{MPa}]$ & 320 & 62 & 1,400 & 53 \\
\hline
\end{tabular}

torn off the substrate. We define the security factor $S_{F}$ to be this maximal weight divided by the weight of our $5 \mathrm{~g}$ mechanism (including the lead weight to simulate the MAV). It indicates the security margin of how well the perching mechanism can support the MAV when perched to the wall.

The mean $S_{F}$ for every of the ten launches per substrate is plotted in Fig. 8 along with its standard deviation. We can observe that for harder substrates the security factor is lower than for softer substrates. However, in the case of the poplar bark, the security factor is one third of the poplar wood despite the fact that it is softer. As we will discuss in more detail later, this effect may be due to the substrate consistence of the poplar bark.

Table 5 Weight budget of the perching mechanism prototype

\begin{tabular}{ll}
\hline Part & Mass [g] \\
\hline Motor & 0.52 \\
10mAh LiPo Battery & 0.58 \\
Gear box & 0.76 \\
Remote control receiver & 0.7 \\
ABS parts & 0.61 \\
Carbon pieces & 0.69 \\
Weight on arms & 0.3 \\
Spring & 0.09 \\
Connection pieces and cables & 0.19 \\
Total mass & 4.61 \\
\hline
\end{tabular}




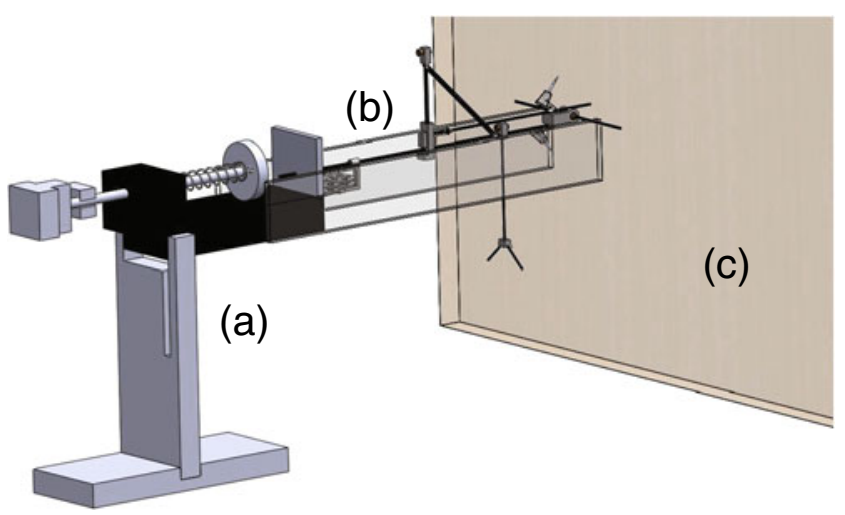

Fig. $7 \mathrm{CAD}$ view of the experimental setup for the perching experiments. The catapult a accelerates the perching mechanism b to a desired impact velocity to the substrate c

\subsection{Impulse balance}

This set of experiments aims at testing if our modeling of the perching mechanism as described in Section 3 is accurate and corresponds to reality. In order to do so, we adjust the weight of our perching mechanism for three different weight configurations, i.e. 2,5 and $8 \mathrm{~g}$, and launch it using the catapult at a velocity of $2 \mathrm{~m} / \mathrm{s}$ towards a wall. The spring constant and the weight of the arms are configured to fit a weight configuration of $5 \mathrm{~g}$ at a flight velocity of $2 \mathrm{~m} / \mathrm{s}$. We test the heavier and the lighter configurations to demonstrate what would happen if the choice of the spring and the masses of the arms would not be appropriate. For every configuration

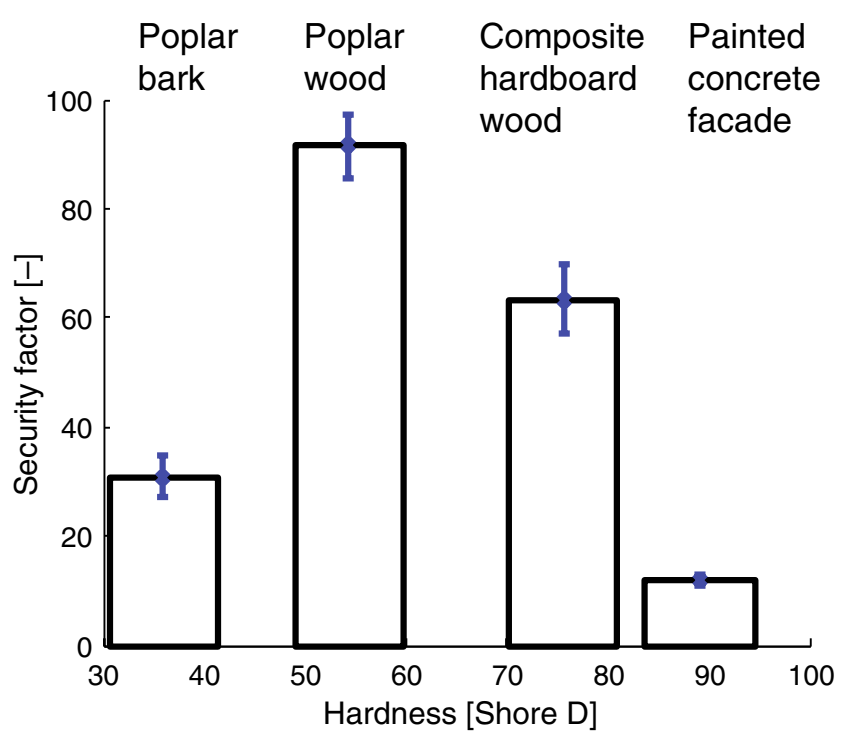

Fig. 8 Security factor for the attachment to the substrate for our system of $5 \mathrm{~g}$ and its standard deviation we perform ten sequential launches. Using a commercially available tracking software [16], we track the position of the front point of the perching mechanism behind the trigger.

The mean and standard deviation of the position versus time is plotted in Fig. 9 for each of the three configurations. This set of experiments is performed using poplar wood as substrate material for the wall. It can be seen that for the $5 \mathrm{~g}$ configuration (Fig. 9b) the oscillations right after impact are almost inexistent, which means that the system is decelerated to zero velocity just in the moment before the needles touch the surface. If we use a slightly too high mass of $8 \mathrm{~g}$ (Fig. 9c), we see that the system is not decelerated sufficiently, crashes into the surface and bounces off. The needles penetrate the substrate at an angle $\psi$ of $104^{\circ}$ instead of $90^{\circ}$ as in the case of the $5 \mathrm{~g}$ configuration. In case that the mass is too low (Fig. 9a), the system remains further away from the surface and the needles penetrate the substrate at an angle of $\psi=73^{\circ}$. Compared to the $8 \mathrm{~g}$ configuration, the mechanism experiences higher frequency oscillations after attachment. The deceleration time $\Delta t$ to zero velocity, which corresponds to the duration between the impact with the surface $t_{1}$ and the contact of the needles with the wall $t_{2}$ has been measured to be $4 \mathrm{~ms}$ for all cases.

These experiments illustrate that the modeling truly does capture the dynamics of the perching mechanism and that the correct choice of the spring and weight of the arms is important to ensure proper perching.

\subsection{Attachment and detachment reliability}

In this set of experiments we evaluate the reliability of the perching mechanism for attachment and detachment on different substrates. We take the same four substrates as before and launch the perching mechanism in its $5 \mathrm{~g}$ configuration towards the wall ten times for every substrate at a velocity of $2 \mathrm{~m} / \mathrm{s}$. The result shows that the attachment to the substrate is successful for all of the 40 sequential launches on all the four substrates. The detachment as well is successful in all cases, but we observe that the effort to detach is different depending on the substrate (Fig. 10). For the painted concrete facade substrate for example, the detachment is achieved already after around one half of the charging cycle, whereby for the softer poplar wood it takes in average 5.6 decharging-recharging cycles to detach. Since one charging cycle takes $1.5 \mathrm{~s}$, the detachment is achieved in average in less than $8.5 \mathrm{~s}$ even for the most difficult of the four substrates. 
Fig. 9 Distance of the perching mechanism from the surface when perching for three different mass configurations. A The entire mechanism has a mass of $2 \mathrm{~g}$, B $5 \mathrm{~g}$ and $\mathbf{C} 8 \mathrm{~g}$. The perching mechanism is designed to fit for the $5 \mathrm{~g}$ configuration (boxed). $\psi$ is the local penetration angle of the needles into the surface
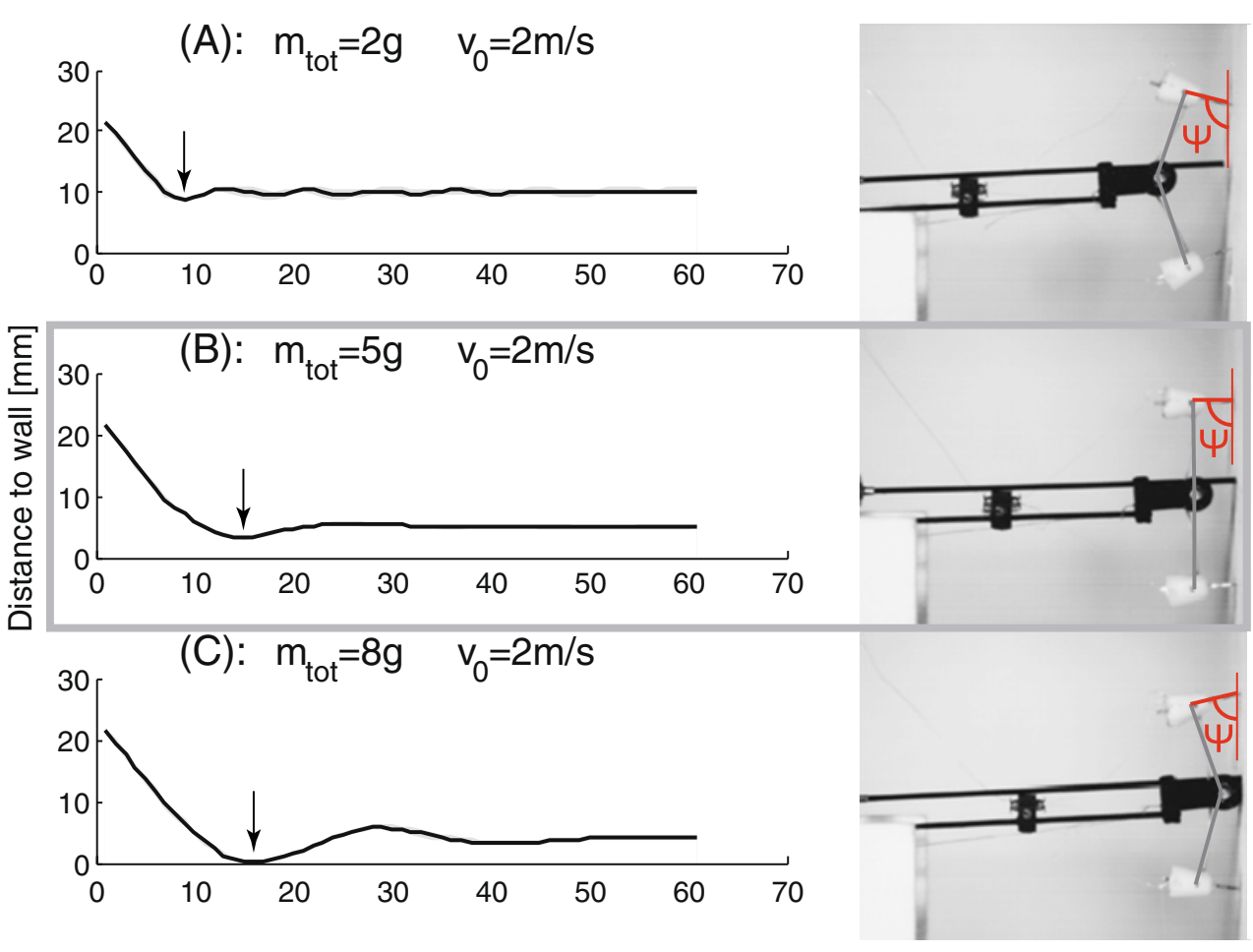

\subsection{Integration with the microglider}

To qualitatively demonstrate that the perching mechanism can successfully be integrated on a MAV, we illustrate a complete perching sequence to a wallpaper wall, a wooden facade of a building and a Marble tree in Fig. 11. In order to balance the microglider, we added

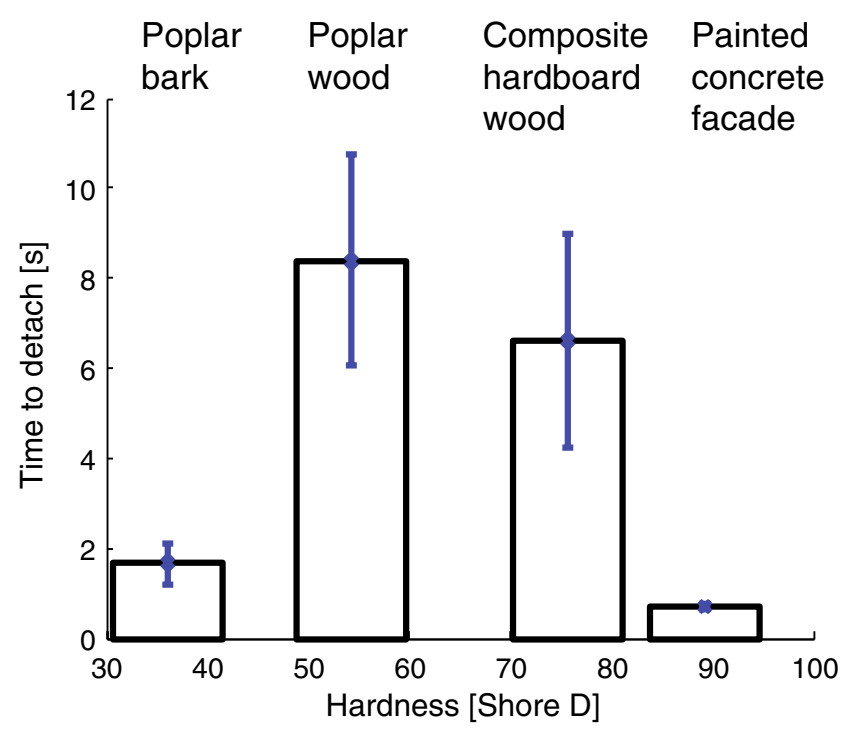

Fig. 10 Time it takes to detach from the four different substrates and its standard deviation small weights to the tail, which reflected in the total weight of the complete system being $6 \mathrm{~g}$. To satisfy the impulse balance as described above, we increased the weight of each arm by 0.05 to $0.2 \mathrm{~g}$ and launched the microglider by hand towards the object. Once the trigger touches the surface, it takes $4 \mathrm{~ms}$ for the snapping movement until the needles stick into the surface. $0.26 \mathrm{~s}$ later, the microglider settles in its final position and is ready to detach. Successful perching and detachment using this integrated microglider to different other walls, facades of buildings and trees can be seen in the accompanying video material. ${ }^{2}$

\section{Discussion}

According to the results derived from the attachment security factor experiments, the attachment force is stronger for softer materials. In the case of the poplar bark, however, the attachment force is only around one third of the poplar wood despite the fact that it is softer. These results suggest that, in general, the hardness of the substrate is a major indication of how well the perching mechanism will stick to it. The somewhat surprising result with poplar wood implies that other

${ }^{2}$ See accompanying video and http://lis.epfl.ch/microglider/ perching.mpg. 
Fig. 11 Perching sequence of the microglider test bed to A a wallpaper wall, $\mathbf{B}$ a wooden facade and $\mathbf{C}$ a Marble tree

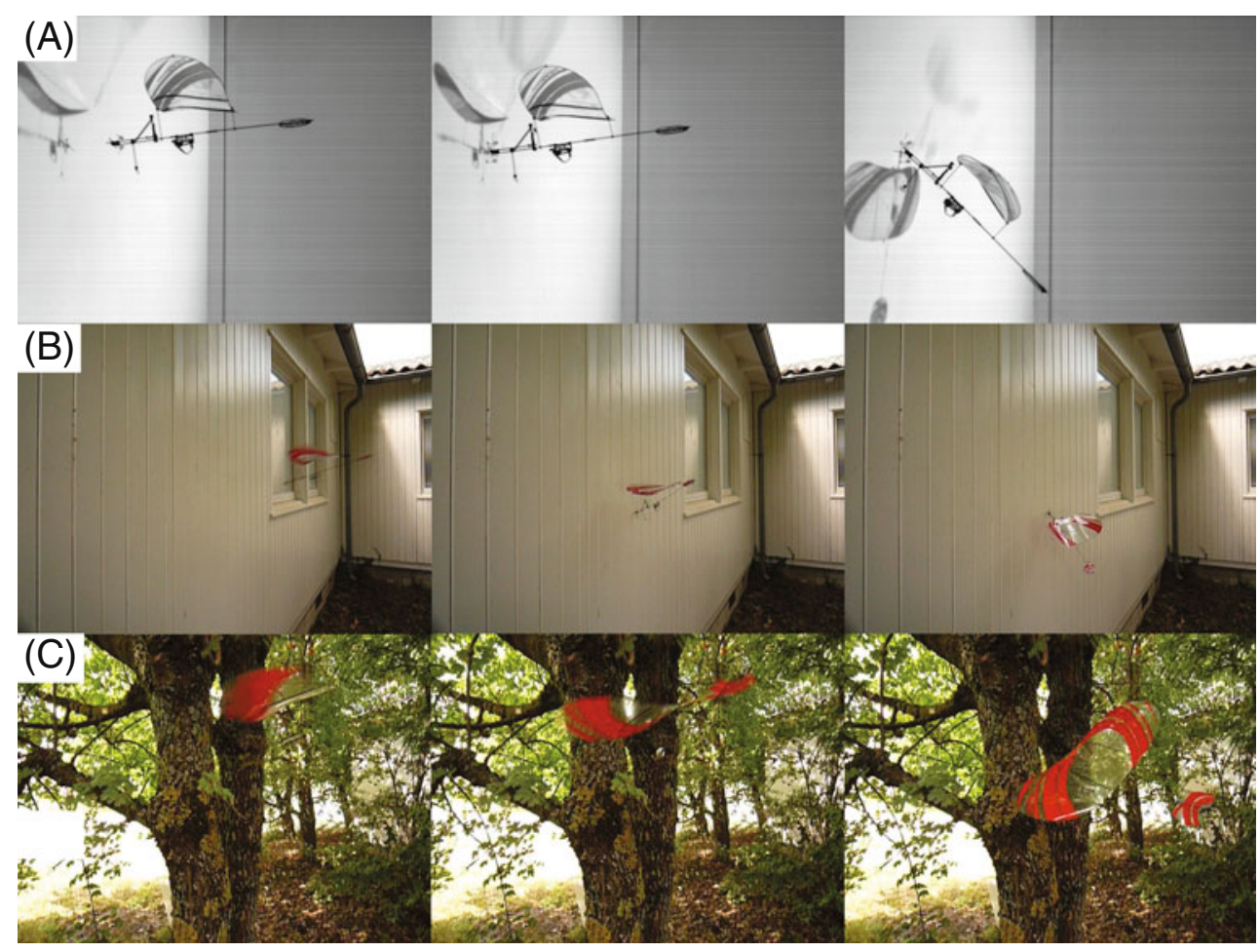

material properties may play a role as well. The needle will enter deeper into softer substrates compared to harder ones and will provide the perching mechanism with more attachment force under the assumption that the only varying parameter is the hardness of the substrate. However, if the substrate is fibrous but of same hardness, it may allow greater attachment force compared to a non-fibrous substrate. On the other extreme, very brittle or porous materials may provide a much lower attachment force. For the experiments performed with our perching mechanism we can note that the security factor for the materials tested ranges between 12 and 91 . These values are very high for an engineering system and are largely sufficient to reliably support the MAV for which the mechanism is designed. For the scope of this article we therefore do not explore in further detail how material properties interact with the needles while perching. Future work could address this question and aim at developing different needle geometries to optimize perching to different substrates.

Also, the cases tested include only substrates with a smooth surface. Depending on the rugosity of the surface, the perching strength may be influenced as the needles may penetrate the substrate locally from different angles, changing the force they can support [29]. Compared to other adhesion methods such as gecko inspired adhesion pads or similar surface attachment techniques, the penetration based adhesion that we use here is still much less sensitive to surface rugosity and can work on very smooth as well as rough surfaces (see accompanying video material which shows successful perching to both, relatively coarse tree barks and smooth wooden plates).

One limitation of the current design is that it cannot attach to very hard surfaces, such as glass or metal walls. A possible extension would therefore be to combine the needle based adhesion with other adhesion methods, e.g. magnets or gecko pads, to enable attachment to these substrates as well. In the animal kingdom, the combination of different adhesion methods is widely used: Wasps, for example, use a combination of van der Waals setae adhesion and spines [8]. In the current perching mechanism, combining it with other adhesion methods would require a partial redesign of the arms. The combination with magnets however can be done very easily without changing the perching mechanism by adding small magnets to the arms. It is noteworthy that one miniature Neodyme-Fer magnet with a diameter of $1.2 \mathrm{~mm}$ and a mass of $0.006 \mathrm{~g}$ can support a weight of up to $20 \mathrm{~g}$ [11]. For very light weight MAVs one could consider magnetizing the needles themselves such that they can support the weight of the MAV when perching to ferromagnetic substrates.

As this article focusses on the development of the perching mechanism and the qualitative demonstration of its successful perching behavior on a microglider test bed, we do not further investigate integration issues with other flying platforms. Nevertheless, we wish to 
provide here a discussion of some aspects that need to be considered if one wants to adapt and use this perching mechanism for another MAV. According to the impulse based model, the perching mechanism can be adapted to any MAV if its mass and flight velocity is known, assuming that the MAV flies forward at a constant flight velocity and that the trigger of the mechanism touches the surface first. It may therefore be best to have the perching mechanism integrated on the most frontal tip of such a flying system. For propelled flight, platforms such as the MicroCeline follow up called Airburr [17] or the swift [23] may be adequate designs because they do not carry the propeller in front.

The main challenge is how to integrate the mechanism on the platform and how to combine it successfully with the global behavior of the MAV. On the microglider in this paper, we attach the mechanism to the fuselage using superglue which is a very convenient and simple way to enable a small MAV with perching capabilities. The perching is achieved without requiring sensing or computation and is, with a success rate of $100 \%$ out of 110 attachment and 40 detachment trials, very robust. This perching mechanism could as well be used for approaches that use dynamic maneuvers to detect the wall and decelerate or position the MAV before attaching to it. In such cases, one or several perching mechanisms could be mounted on the ventral part or on the wings of the MAV. In our microglider with a mass of $6 \mathrm{~g}$, the perching mechanism represents with $4.6 \mathrm{~g}$ a significant fraction of the entire system mass. For larger, or other types of MAVs such as for example a quadrotor-like hovering platform, it could be possible to integrate several perching mechanisms on its outer periphery, since the mechanism weights only a few grams. The challenge in this case would be to ensure that the MAV approaches the wall at a sufficiently steep angle and that only one perching mechanism is released at once.

The cases that we tested in this article are performed perpendicular to the wall in both pitch and yaw. The attachment propensity may decrease with the angle at which the MAV flies to the wall such that below a certain threshold, attachment may no more be feasible. Based on the experience with the perching mechanism presented here, the limit for it to attach is in the order of $\pm 45^{\circ}$ in pitch and $\pm 30^{\circ}$ in yaw. This may nevertheless vary depending on the substrate, the flight velocity and the mass of the MAV. A systematic characterization thereof is beyond the scope of this paper. One possible solution how to ensure that the perching mechanism faces the wall perpendicularly in pitch may be to add a hinge between the mechanism and the MAV and add a small weight to the mechanism that would, due to gravity, keep the mechanism oriented vertically.

Depending on the MAV where the perching mechanism is integrated, the take-off after detachment from the wall may be a challenge as well and require a coordination of detachment and the propulsion of the MAV. For this, the support rod (Fig. 5a(k)) could be adapted in shape and length to keep the MAV in a favorable position to ease the transition to flight after detachment. A possibility would be to use auto-stable MAV platforms that, after detachment from the wall, self-stabilize and navigate away from the wall in flight. Another way would be to jump off the wall using a small jumping mechanism e.g. the one presented in [18] with a weight of only $5.7 \mathrm{~g}$. Using the combination of gliding, perching and jumping off the wall has been as well described within the Self Deploying Microglider project [19, 21].

For MAVs that fly very fast or are very heavy, one needs to keep in mind that the kinetic energy of a moving object is defined as $E_{k i n}=(1 / 2) \cdot m_{\text {mav }} v_{\text {mav }}^{2}$. Assuming that the deceleration is constant, we can express the force during impact as $F_{\text {impact }}=E_{\text {kin }} / \Delta s$, where $\Delta s$ is the deceleration distance. This means that the force acting on the MAV is linear proportional to its mass, quadratically proportional to its flight velocity and inversely proportional to the deceleration distance. For our case of a $6 \mathrm{~g}$ microglider flying at $2 \mathrm{~m} / \mathrm{s}$, and a deceleration time $\Delta t$ of $4 \mathrm{~ms}$, the forces acting on the structure are $F_{\text {impact }}=m \cdot v_{\text {mav }} / \Delta t=2.5 \mathrm{~N}$, which is acceptable. For comparison, a dart like design with a penetration depth of $0.5 \mathrm{~mm}$ would lead to a very high and potentially hazardous force of $48 \mathrm{~N}$. We can also assume that the deceleration distance is proportional to the arm length $l_{s}$ of the perching mechanism. These basic scaling laws imply that for very fast and heavy MAVs, the arms of the perching mechanism need to be dimensioned proportional to $m v_{\text {mav }}^{2} / \Delta s$ if the forces acting on the structure of the MAV should be constant.

Future work could include the integration of the perching mechanism on different MAVs and a more detailed assessment of the overall performance of perching enabled MAVs. Also, future work could address the combination of the penetration based clinging with other adhesion methods to enable it to attach to a larger variety of substrates.

\section{Conclusion}

In this article we presented the development of a simple and lightweight perching mechanism that can attach to and detach from trees and facades of buildings. We 
showed that it can reliably, with a success rate of $100 \%$, perch to four different substrates with a security factor between 12 and 91 . Based on the model presented in this paper, the perching mechanism can be dimensioned to be used on different other MAVs as well, assuming that their mass and flight velocity is known. However, the integration on the platforms and the overall system performance needs to be considered carefully case by case. Summarizing, we present a working perching mechanism module that enables a MAV to perform repetitive head-first perching to vertical man-made and natural structures, while limiting the impact forces acting on the MAV. Such a perching MAV may be very useful for the deployment of sensor networks for a variety of applications.

Acknowledgements We would like to thank Jean-Christophe Zufferey from the Laboratory of Intelligent Systems at EPFL for proofreading the article and the constructive feedback on the experiments. As well, we thank the Atelier d'électromécanique (AEM) for the production of the parts. This project is funded by the EPFL, by the Swiss National Science Foundation, grant number 200020-116149 and by the European project Swarmanoid of the Future and Emergent Technology division.

\section{References}

1. Anderson ML, Perry CJ, Hua BM, Olsen DS, Parcus JR, Pederson KM, Jensen DD (2009) The sticky-pad plane and other innovative concepts for perching uavs. In: Proceedings of the 47th AIAA aerospace sciences meeting

2. Asbeck AT, Kim S, Cutkosky MR, Provancher WR, Lanzetta M (2006) Scaling hard vertical surfaces with compliant microspine arrays. Int J Rob Res 25(12):1165

3. Autumn K, Sitti M, Liang YA, Peattie AM, Hansen WR, Sponberg S, Kenny TW, Fearing R, Israelachvili JN, Full RJ (2002) Evidence for van der waals adhesion in gecko setae. Proc Natl Acad Sci 99(19):12252-12256

4. Bayraktar S, Feron E (2008) Experiments with small helicopter automated landings at unusual attitudes. Arxiv preprint arXiv:0709.1744

5. Cortes J, Martinez S, Karatas T, Bullo F (2004) Coverage control for mobile sensing networks. IEEE Trans Robot Autom 20(2):243-255

6. Cory R, Tedrake R (2008) Experiments in fixed-wing uav perching. In: AIAA conference on guidance, navigation, and control

7. Daltorio KA, Horchler AD, Gorb SN, Ritzmann RE, Quinn RD (2005) A small wall-walking robot with compliant, adhesive feet. In: IEEE/RSJ international conference on intelligent robots and systems, pp 3648-3653

8. Frantsevich L, Gorb S (2004) Structure and mechanics of the tarsal chain in the hornet, vespa crabro (hymenoptera: Vespidae): implications on the attachment mechanism. Arthropod Struct Develop 33(1):77-89. Attachment Systems of Arthropods
9. Gao H, Yao H (2004) Shape insensitive optimal adhesion of nanoscale fibrillar structures. Proc Natl Acad Sci 101(21):7851-7856

10. Gorb SN (2008) Biological attachment devices: exploring nature's diversity for biomimetics. Phil Trans R Soc A Math Phys Eng Sci 366(1870):1557

11. http://www.didel.ch Didel SA (2008)

12. http://www.durovis.ch/ Durovis steel torsion springs (2009)

13. http://www.falconmodels.uk.com Falcom mk iv $1.6 \mathrm{~g}$ servo (2009)

14. http://www.fastecimaging.com Troubel shooter 1000 high speed camera (2007)

15. http://www.funtech.com Dimension elite 3d printer (2008)

16. http://www.xcitex.com Proanalyst motion analysis software (2008)

17. Klaptocz A, Boutinard Rouelle G, Briod A, Zufferey JC, Floreano D (2010) An indoor flying platform with collision robustness and self-recovery. In: IEEE/RSJ international conference on robotics and automation (to appear)

18. Kovac M, Fuchs M, Guignard A, Zufferey J-C, Floreano D (2008) A miniature $7 \mathrm{~g}$ jumping robot. In: IEEE int conf robot autom, pp 373-378

19. Kovac M, Fuchs M, Savioz G, Guignard A, Nicoud J-D, Zufferey J-C, Floreano D (2007) Self deploying microglider. In: Flying insects and robots symposium

20. Kovac M, Guignard A, Nicoud J-D, Zufferey J-C, Floreano D (2007) A 1.5 g SMA-actuated microglider looking for the light. In: IEEE int conf robot autom, pp 367-372

21. Kovac M, Zufferey JC, Floreano D (2009) Towards a selfdeploying and gliding robot. In: Floreano D, Zufferey J-C, Srinivasan MV, Ellington C (eds) Flying insects and robots, chapter 19. Springer, Heidelberg

22. La Rosa G, Messina M, Muscato G, Sinatra R (2002) A lowcost lightweight climbing robot for the inspection of vertical surfaces. Mechatronics 12(1):71-96

23. Leven S, Zufferey J-C, Floreano D (2007) A simple and robust fixed-wing platform for outdoor flying robot experiments. In: International symposium on flying insects and robots, pp 69-70

24. Lussier-Desbiens A, Cutkosky MR (2010) Landing and perching on vertical surfaces with microspines for small unmanned air vehicles. J Intell Robot Syst 57(1):313-327

25. Mainwaring A, Culler D, Polastre J, Szewczyk R, Anderson J (2002) Wireless sensor networks for habitat monitoring. In: Proceedings of the 1st ACM international workshop on wireless sensor networks and applications

26. Murphy MP, Sitti M (2007) Waalbot: an agile smallscale wall-climbing robot utilizing dry elastomer adhesives. IEEE/ASME Trans Mechatron 12(3):330-338

27. Nicoud J-D, Zufferey J-C (2002) Toward indoor flying robots. In: IEEE/RSJ international conference on robots and systems (IROS'02). Lausanne, pp 787-792

28. Prahlad H, Pelrine R, Stanford S, Marlow J, Kornbluh R (2008) Electroadhesive robots-wall climbing robots enabled by a novel, robust, and electrically controllable adhesion technology. In: Robotics and automation, 2008, IEEE international conference on, pp 3028-3033

29. Provancher WR, Clark JE, Geisler B, Cutkosky MR (2004) Towards pentration-based clawed climbing. In: Proceedings of the 7 th international conference on climbing and walking robots (CLAWAR 2004), vol 1, pp 22-24

30. Qian Z, Zhao Y, Fu Z (2006) Development of wall-climbing robots with sliding suction cups. In: IEEE/RSJ international conference on intelligent robots and systems, pp 3417-3422 
31. Roberts JF, Zufferey JC, Floreano D (2008) Energy management for indoor hovering robots. In: IEEE/RSJ international conference on intelligent robots and systems (IROS2008), pp 1242-1247

32. Santos D, Heyneman B, Kim S, Esparza N, Cutkosky MR (2008) Gecko-inspired climbing behaviors on vertical and overhanging surfaces. In: Robotics and automation, 2008, IEEE international conference on, pp 1125-1131

33. Shen W, Gu J, Shen Y (2006) Permanent magnetic system design for the wall-climbing robot. Applied Bionics and Biomechanics 3(3):151-159

34. Ullman DG (2002) The mechanical design process. McGrawHill, New York
35. Wickenheiser AM, Garcia E (2008) Optimization of perching maneuvers through vehicle morphing. J Guid Control Dyn 31(4):815-823

36. Wile GD, Daltorio KA, Diller ED, Palmer LR, Gorb SN, Ritzmann RE, Quinn RD (2008) Screenbot: walking inverted using distributed inward gripping. In: Robotics and automation, IEEE international conference on, pp 1513-1518

37. Wright K, Lind R (2007) Investigating sensor emplacement on vertical surfaces for a biologically-inspired morphing design from bats. In: AIAA atmospheric flight mechanics conference and exhibit

38. Zufferey J-C, Floreano D (2006) Fly-inspired visual steering of an ultralight indoor aircraft. IEEE Trans Robot 22:137-146 